\title{
À quoi ressemblent les classes moyennes en Côte d'Ivoire aujourd'hui? Continuités historiques et enjeux renouvelés d'un ensemble hétérogène
}

\author{
Jean-Philippe Berrou, Dominique Darbon, Anne Bekelynck, Christian \\ Bouquet, Matthieu Clément, François Combarnous \& Éric Rougier
}

To cite this article: Jean-Philippe Berrou, Dominique Darbon, Anne Bekelynck, Christian Bouquet, Matthieu Clément, François Combarnous \& Éric Rougier (2020): À quoi ressemblent les classes moyennes en Côte d'Ivoire aujourd'hui? Continuités historiques et enjeux renouvelés d'un ensemble hétérogène, Canadian Journal of Development Studies / Revue canadienne d'études du développement, DOI: 10.1080/02255189.2020.1711365

To link to this article: https://doi.org/10.1080/02255189.2020.1711365

View supplementary material $₫$

Published online: 25 Feb 2020.

Submit your article to this journal

a

View related articles $\asymp$

View Crossmark data $\asymp$ 


\title{
À quoi ressemblent les classes moyennes en Côte d'Ivoire aujourd'hui? Continuités historiques et enjeux renouvelés d'un ensemble hétérogène
}

\author{
Jean-Philippe Berrou ${ }^{a}$, Dominique Darbon ${ }^{a}$, Anne Bekelynck $^{b}$, Christian Bouquet $^{a}$, \\ Matthieu Clément ${ }^{c}$, François Combarnous ${ }^{c}$ et Éric Rougier ${ }^{c}$ \\ aLes Afriques dans le monde (LAM), Centre national de la recherche scientifique (CNRS), Sciences Po \\ Bordeaux, Bordeaux, France; ${ }^{\mathrm{B}} \mathrm{PAC}-\mathrm{Cl}$, Agence nationale de recherche sur le sida et les hépatites virales \\ (ANRS), Côte d'Ivoire, Abidjan, Côte d'Ivoire; 'Groupe de recherche en économie théorique et appliquée \\ (GREThA), Centre national de la recherche scientifique (CNRS), Université de Bordeaux, Bordeaux, France
}

\begin{abstract}
RÉSUMÉ
L'essor des classes moyennes en Côte d'Ivoire constitue un enjeu économique et politique central. Cet article analyse la composition de cette catégorie de revenus et son inscription dans les régimes de croissance du pays. En s'appuyant sur une méthodologie combinant des données d'enquêtes quantitatives et qualitatives, nos analyses montrent que les classes moyennes ivoiriennes représentent un ensemble limité et très hétérogène de la population, regroupant à la fois des piliers historiques de la période faste des années 1960-1970 et les traces des ajustements structurels de même que celles des crises qui suivirent. Le " secteur informel » de l'économie se révèle être une voie majeure d'ascension sociale.
\end{abstract}

\section{ABSTRACT}

The rise of the middle classes in the Ivory Coast represents a key economic and political issue. This article analyses the composition of this income category as well as its role in the country's growth regime. Using data from both quantitative and qualitative surveys, our analyses show that the Ivory Coast's middle classes represent a limited and very heterogeneous subset of the population. They bring together the social and historic pillars of the 1960s and 1970 s boom years and the traces of structural adjustments as well as those of subsequent crises. It turns out that the "informal" sector is a major pathway to social advancement.

\section{CHEMINEMENT DE} L'ARTICLE

Reçu le 21 mai 2018

Accepté le 9 octobre 2019

\section{MOTS CIÉS}

Côte d'Ivoire; classe moyenne; stratification sociale; régime de croissance; méthodes de classification

\section{Introduction}

En 2011, la Banque africaine de développement (BAD) publie le Market Brief intitulé « The Middle of the Pyramid: Dynamics of the Middle Class in Africa » et annonce que près d'un tiers la population du continent africain appartient à la classe moyenne. Depuis, les magazines économiques et les cabinets internationaux de marketing lui ont emboîté le pas et ont fait du thème de l'émergence des classes moyennes un enjeu central du futur de l'Afrique. La 
Côte d'Ivoire n'échappe pas à cette euphorie. Dans le contexte d'un nouveau cycle de forte croissance économique depuis la fin de la crise postélectorale de 2010, la question de l'essor des classes moyennes ivoiriennes fait l'objet d'un vif intérêt de la part des opérateurs privés et est largement relayée par la presse internationale et nationale.

L'essor des classes moyennes est présenté dans la littérature universitaire comme déterminant dans le processus de développement d'un pays. Il permet l'accroissement des dépenses de consommation et l'expansion du marché domestique et, par là même, la croissance du PIB. Il contribue à la diversification de la demande sociale et de la consommation de biens et services (Banerjee et Duflo 2008). Il modifie la structure sociale et révèle le potentiel de mobilité ascendante dans les strates de la société. Il redistribuerait enfin les enjeux politiques en rendant possible une stabilisation politique autour d'une masse importante de la population (Easterly 2001) ou, à l'inverse, pourrait augmenter le risque de déstabilisation politique en diversifiant et en renforçant les sources de conflits et de revendications (Huntington 1968).

Multidisciplinaire par essence, le concept de « classe moyenne » reste encore très largement controversé au sein de la littérature des sciences sociales, tant dans sa définition que dans son utilisation. Différentes approches ont été mobilisées pour analyser ce phénomène. L'approche économique utilise principalement un critère de revenu, de consommation ou de richesse afin de distinguer les ménages ou individus de la classe moyenne de ceux considérés comme pauvres ou riches. Les approches plus sociologique et politique se fondent sur l'observation du statut social (éducation, catégorie socioprofessionnelle [CSP], type d'emploi), du prestige ou de l'identité de classe (préférences, aspirations) et du pouvoir effectivement détenu. Les approches managériales reposent quant à elles davantage sur la caractérisation des habitudes de consommation et de leur transformation. Il résulte de cette grande diversité des approches un usage souvent peu précautionneux et parfois confus du terme "classe moyenne ». ${ }^{1}$

En Côte d'Ivoire, la notion de classe moyenne est quasi absente des documents officiels alors même qu'elle est très présente dans la presse économique et généraliste. Un battage médiatique autour des classes moyennes est ainsi entretenu par les acteurs économiques des marchés de la consommation et de la publicité. Les deux études de marché récentes sur la classe moyenne abidjanaise conduites par IPSOS pour le compte de la CFAO (2015) et de UCT-UNILEVER (2017) en témoignent. Les ouvertures, depuis 2015, de grands centres commerciaux à Abidjan (Playce Marcory, Cocody-Riviera Palmeraie) sont également une illustration de l'engouement du secteur privé pour l'essor présupposé de ces classes moyennes (Nallet 2018). En Côte d'Ivoire, comme dans le reste de l'Afrique, on repère ainsi la classe moyenne principalement par un mouvement global d'accroissement des revenus et d'augmentation des capacités de consommation. Pour autant, ces dynamiques tendent à masquer la profonde hétérogénéité des situations sociales concernées par ce processus. Des travaux récents réalisés dans différents pays africains ont ainsi discuté de la pertinence de l'utilisation du terme " classe moyenne » dans ces contextes (Darbon 2012; Kroeker, O’Kane, et Scharrer 2018; Melber 2016).

Pourtant, la question des classes moyennes en Côte d'Ivoire intéresse fortement tant la communauté scientifique que les décideurs politiques et économiques, ses enjeux allant bien au-delà de la seule question de l'expansion d'un marché de consommation. Ces derniers sont tout autant sociaux (cohésion sociale dans un contexte post-crise de réconciliation nationale), économiques (développement d'un marché interne nécessaire aux objectifs de diversification de l'économie) que politiques (légitimité et stabilité politique 
dans un contexte de fortes attentes sociales). Le gouvernement du président A. Ouattara a par exemple fait de la croissance économique et de son ruissellement un instrument majeur du processus de réconciliation nationale et de reconstruction de la cohésion sociale (Akindès 2018). En outre, la situation de la Côte d'Ivoire est d'autant plus intéressante que la structuration des groupes de revenu intermédiaire s'y inscrit dans l'histoire longue, les classes moyennes ayant représenté une part significative de la population dans la période dite du « miracle » économique des années 1960-1970, avant que ce mouvement de transformation de la stratification sociale ne s'interrompe à la suite de près de trente années d'instabilités socioéconomiques et politiques.

Cet article se propose d'analyser comment se compose cette catégorie de revenu intermédiaire dans la société ivoirienne contemporaine et les enjeux socioéconomiques et politiques qui lui sont associés. À cet effet, il poursuit trois objectifs principaux : (1) analyser les processus de stratification sociale et d'évolution des catégories intermédiaires de revenus dans leur articulation avec le régime de croissance ivoirien depuis les indépendances; (2) proposer une méthode d'identification des classes moyennes ivoiriennes contemporaines permettant notamment d'en souligner l'hétérogénéité; et (3) analyser les caractéristiques et les comportements de ces classes moyennes et les enjeux que cela soulève pour le pays. D'un point de vue méthodologique, nous adoptons une démarche séquentielle et mixte en combinant analyse de la littérature universitaire et institutionnelle, analyse de données quantitatives (données de l'Enquête nationale sur le niveau de vie des ménages [ENV 2015]) et analyse de données qualitatives (entretiens semi-directifs auprès d'une trentaine de ménages).

Dans ce qui suit, la trajectoire de stratification sociale et de formation des classes moyennes en Côte d'Ivoire est d'abord mise en perspective, notamment au regard de la séquence des régimes de croissance économique suivie par le pays (première partie). Ensuite, nous mettons en œuvre une méthodologie d'identification et de caractérisation de la classe moyenne ivoirienne contemporaine dans sa globalité (deuxième partie) avant d'analyser plus finement son hétérogénéité et les différences potentielles de comportements et d'aspirations propres à ce groupe (troisième partie). Nous terminons par une discussion sur les enjeux socioéconomiques que nos résultats soulèvent pour le développement économique du pays, avant de conclure.

\section{Régime de croissance et stratification sociale : émergence, déclin et renaissance des classes moyennes ivoiriennes?}

Le modèle de croissance ivoirien et la trajectoire socioéconomique du pays ont été et restent encore profondément conditionnés par l'évolution et les modalités de gestion et de redistribution de sa "rente forêt " (bois, café, cacao, hévéa, etc.). Le graphique A1 (annexé en ligne), qui montre très bien la forte corrélation entre l'évolution de la valeur des exportations de cacao et de café et celle du PIB par habitant, permet également de reconnaître cinq grandes phases historiques utiles pour analyser et comprendre les processus de stratification sociale dans le pays.

\section{L'émergence ancienne des catégories de revenu intermédiaire}

Au lendemain de son indépendance, la Côte d'Ivoire a connu une période faste de près de 20 ans, avec une croissance annuelle moyenne de son PIB réel par habitant de près de 
4 pour cent (Cogneau et Mesplé-Somps 2003). Cette croissance est portée par un environnement international favorable ("boom » des prix du café et du cacao) dont profite le développement extensif des agricultures d'exportation. La politique d'encadrement des prix à travers l'organisme de la Caistab (Caisse de stabilisation et de soutien des prix des produits agricoles) permet d'assurer aux planteurs des revenus conséquents et relativement stables. Le compromis politico-économique mis en place par Houphouët-Boigny repose alors largement sur une redistribution stratégique de la rente agricole jusqu'aux niveaux les plus bas de la hiérarchie sociale (Fauré et Médard 1982). Les surplus agricoles considérables permettent de financer une politique volontariste de grands investissements dans les infrastructures, la santé, l'éducation et le logement, ainsi qu'une politique salariale et de recrutement avantageuse dans la fonction publique. Aux côtés de la bourgeoisie d'État, le miracle économique ivoirien entraîne alors dans son sillage ce que certains auteurs qualifient déjà de «classes moyennes » et que le langage populaire désigne par l'expression "les en-haut des en-bas et les en-bas des en-haut " (Savina et coll. 1985). Au-delà des dizaines de milliers de planteurs qui en font partie, ces groupes intermédiaires ont aussi appuyé leur enrichissement sur le rendement de leur investissement éducatif, en s'arrogeant les emplois salariés les plus attirants des secteurs publics et privés urbains en expansion. Savina et coll. (1985) estiment que les classes moyennes représentent en 1978 à Abidjan environ 345000 personnes, soit 28 pour cent de la population de la métropole.

\section{Le long déclin des classes moyennes (1980-2010)}

À partir des années 1980, la Côte d'Ivoire connaît une sévère crise économique et financière (crise de la dette). Le pays entre alors dans une phase marquée par la succession de plans d'ajustements structurels impliquant diverses mesures de restrictions budgétaires et de restructuration du secteur public. Cette période marque un coup d'arrêt dans le processus d'émergence des classes moyennes qui se retrouvent frontalement " conjoncturées » (Le Pape 1997). Avec la compression des effectifs de la fonction publique, la réduction des salaires et la destruction de l'emploi salarié dans le secteur privé formel, on assiste à une prolifération des activités informelles. Il en résulte une expansion considérable de la pauvreté (graphique A2, annexé en ligne).

La période qui suit le décès du président Houphouët-Boigny en 1993 est marquée par une libéralisation accrue de l'économie, la dévaluation du franc CFA en 1994 et la montée des tensions politiques dans un contexte de "guerre » de succession. Avec la dévaluation, le pays renoue avec la croissance économique, mais celle-ci n'aura pas les effets de ruissellement escomptés (Grimm, Guénard, et Mesplé-Somps 2002). L'élite dominante qui détient le foncier rural ou du capital dans les grandes entreprises - notamment étrangères et exportatrices - va, à la faveur de la dévaluation, bénéficier d'une forte appréciation de ses avoirs extérieurs (Contamin et Memel Foté 1997). Les autres catégories sociales, et notamment les classes moyennes, ne disposent pas des mêmes chances et voient leur situation se dégrader. Les inégalités de revenus augmentent fortement à Abidjan (le coefficient de GINI passe de 0,49 à 0,53 ) comme en milieu rural (de 0,42 à 0,49) (Cogneau et MespléSomps 2003). L'évidence d'une rupture s'impose et cette situation ne fait qu'accentuer les tensions sociales et fragiliser les équilibres politiques sur fond d'instrumentalisation ethnonationaliste à travers le concept d' "ivoirité ». 
Le coup d’État du Général Gueï en 1999 est le point de départ d’une longue période d'instabilités socioéconomiques et politiques en Côte d'Ivoire, qui ne prendra fin qu'en 2011. Durant cette période, l'économie est paralysée et on assiste à une paupérisation massive de la société ivoirienne. Entre 2002 et 2008, l'incidence de la pauvreté passe de 38,4 pour cent à 48,9 pour cent (graphique A2). Les groupes qui étaient assimilables aux classes moyennes sont en voie de disparition.

\section{Le renouveau économique de la Côte d'lvoire signe-t-il le retour des classes moyennes?}

Depuis 2011 et la fin de la dernière crise postélectorale, la Côte d'Ivoire a renoué avec une croissance économique spectaculaire avoisinant les 8 pour cent par an en moyenne (OCDE 2016). Fait notable, c'est principalement la demande intérieure qui joue un rôle central dans cette dynamique, avec, notamment, des plans publics d'investissement ambitieux et des mesures politiques volontaristes de soutien au revenu des ménages. Le PIB par habitant croît ainsi durant la période à un rythme proche des 6 pour cent par an. Ce climat favorable a porté sur le devant de la scène le thème de la réémergence des classes moyennes ivoiriennes. Pour autant, il faut rappeler que cette forte croissance économique est restée peu inclusive. Le marché de l'emploi en Côte d'Ivoire est encore dominé par le « secteur informel » qui assure près de 90 pour cent de l'emploi (ENV 2015). L'incidence de la pauvreté, au seuil national de pauvreté (soit une dépense de consommation journalière inférieure à 737 FCFA), était de 46,3 pour cent en 2015 et les inégalités de revenus se maintiennent à un niveau élevé (Cogneau, Czajka et Houngbedji 2017; ENV 2015).

C'est dans ce contexte qu'il faut resituer les estimations du poids de la classe moyenne en Côte d'Ivoire. Selon sa définition (la classe moyenne se situant entre 2 \$ et 20 \$ PPA de dépenses de consommation par tête et par jour), la BAD (2011) estimait qu'elle représentait 37,1 pour cent de la population en 2010. Pour la même année, avec des données actualisées, Ncumbe et Lufumpa (2015) estimaient qu'elle représentait 55 pour cent de la population, en précisant toutefois que plus de 60 pour cent de ce groupe relevait de la " classe flottante » (entre 2 \$ et 4 \$) et seuls 5 pour cent de la "classe moyenne supérieure » (entre 10 \$ et $20 \$$ ). Comme on le voit avec ces chiffres anciens, il existe un enjeu de connaissances important lié à la quantification et à la caractérisation du groupe de revenu intermédiaire en Côte d'Ivoire.

\section{Données et méthodologie d'identification et de caractérisation des classes moyennes ivoiriennes}

\section{Une méthodologie quantitative séquentielle}

Concernant les aspects quantitatifs, les données mobilisées sont celles issues de l'enquête ENV (Enquête niveau de vie des ménages) conduite par l'INS (Institut national de la statistique) en 2015. L'ENV 2015 prend pour base d'échantillonnage le recensement général de la population de 2014. L'enquête a été conduite par l'INS entre février et mars 2015 et couvre 12900 ménages représentatifs de l'ensemble des ménages africains résidant en Côte d'Ivoire (ENV 2015). Comme le soulignent Cogneau, Czajka, et Houngbedji (2017), certains biais de représentativité persistent concernant les résidents de nationalités française 
et libanaise qui ne sont pas intégrés. De même, la taille moyenne des ménages estimée dans l'ENV 2015 apparaît plus faible (3,6 personnes) que dans le recensement de la population $(5,4)$. Il faut conserver à l'esprit ces biais et faire preuve de prudence dans l'interprétation de nos résultats statistiques. L'enquête s'inscrit par ailleurs dans la continuité des précédentes vagues de 2002 et 2008 sur le plan de la structure du questionnaire adapté des «Living Standards Measurement Surveys » (LSMS) de la Banque mondiale. ${ }^{2}$

Ces données quantitatives sont utilisées pour délimiter une classe moyenne de revenu au sein de la population, révéler son hétérogénéité en distinguant différents sous-groupes homogènes et en établir les principales caractéristiques. La méthode adoptée est séquentielle et suit, dans les grandes lignes, la démarche adoptée par Bonnefond, Clément, et Combarnous (2015). Elle combine une approche économique basée sur le revenu et une approche plus sociologique et multidimensionnelle mobilisant des informations sur l'emploi et l'éducation (Thurlow, Resnick, et Ubogu 2015).

Nous adoptons tout d'abord une approche " pécuniaire " par le revenu pour délimiter un « ensemble du milieu » de la population. L'objectif n'est pas tant ici de déterminer précisément la taille de la classe moyenne (compte tenu de l'absence de consensus sur le choix de l'intervalle pécuniaire) que de délimiter un ensemble d'individus situés au milieu de la distribution du niveau de vie dans le pays (c.-à-d. les « gens du milieu »). Tout d'abord, nous avons opté pour une mesure pécuniaire basée sur le revenu, plutôt que sur la dépense de consommation. L'indicateur retenu est le revenu des ménages par tête et intègre toutes les composantes habituelles du revenu (revenus du travail formel et informel, revenus du patrimoine, transferts publics et privés, etc.). ${ }^{3}$ Ensuite, il nous faut définir l'intervalle de revenu permettant de délimiter les individus qui composent la classe moyenne. Trois grandes approches existent à cet effet. L'approche absolue fondée sur des seuils internationaux exprimés en dollars PPA (parité des pouvoirs d'achat) est avant tout consacrée aux comparaisons internationales. Le seuil international de pauvreté de 2 \$ s'est ici rapidement imposé comme borne inférieure de l'intervalle de revenu. C'est l'optique retenue par la BAD (2011) avec un intervalle de 2 \$-20 \$. La classe moyenne est alors censée commencer là où la pauvreté se termine. L'approche relative est plus contextuelle et consiste à définir cette classe comme la population qui se situe au milieu de la distribution du revenu. Les intervalles sont alors principalement construits à partir du revenu médian (75 \%-125\% du revenu médian, $50 \%-150 \%$ du revenu médian, etc.). Une troisième approche, dite approche mixte, consiste à combiner une borne inférieure absolue et une borne supérieure relative (Birdsall 2010). L'analyse de la distribution du revenu en Côte d'Ivoire nous donnera des " guides » pour le choix d'un intervalle pécuniaire pertinent.

Ensuite, afin d'explorer l'hétérogénéité de cette classe moyenne de revenu, nous mettons en œuvre une procédure de classification mixte issue de la statistique exploratoire multidimensionnelle sur plusieurs variables, décrivant les statuts occupationnels et éducatifs des ménages de l'échantillon considéré. À cet effet, cinq variables liées à l'éducation et à l'emploi ont été retenues : (1) le niveau de formation le plus élevé atteint par le chef du ménage; (2) la catégorie socioprofessionnelle du chef du ménage; (3) le statut dans l'emploi du chef du ménage; (4) le type d'employeur ou de secteur institutionnel du chef du ménage; et (5) la pluriactivité du chef du ménage. Sur la base de ces cinq variables, on effectue avec la procédure retenue une classification d'un grand ensemble d'individus caractérisés par leurs premières coordonnées factorielles créées par une procédure initiale 
d'analyse factorielle (ici, une analyse des correspondances multiples). Une première classification est obtenue par croisement de plusieurs partitions de base construites autour de centres mobiles, puis les classes stables ainsi formées sont agrégées par une méthode de classification hiérarchique. ${ }^{4}$ Puis, nous proposons une classification en groupes homogènes et clairement distincts les uns des autres.

\section{Une méthode mixte : l'apport des entretiens qualitatifs}

Les données qualitatives sont issues d'entretiens semi-dirigés conduits sur les périodes de mai-juin et octobre-novembre 2017 auprès de 31 ménages représentant la diversité des classes moyennes identifiées lors de l'analyse quantitative (voir le tableau A1, annexé en ligne). À cet effet, les ménages ont été sélectionnés selon la méthode des quotas et les entretiens ont été menés en deux lieux distincts : la capitale économique du pays (Abidjan) et une zone plus rurale (Soubré). La grille d'entretien thématique visait à couvrir les thèmes jugés importants pour l'étude et à faciliter la comparaison entre les discours collectés. ${ }^{5}$ L'objectif de ces entretiens était de compléter les informations produites par l'analyse quantitative des données. Il s'agissait de parvenir à une meilleure compréhension « de l'intérieur » des représentations, attitudes, motivations et pratiques d'un groupe social particulier, la classe moyenne, et de ses différentes composantes.

\section{Les classes moyennes en Côte d'Ivoire en 2015 : un ensemble limité et hétérogène}

\section{Les gens du milieu de la distribution des revenus}

Le Graphique 1 représente la distribution (fonction kernel) des revenus mensuels par tête des ménages ivoiriens en 2015, ainsi que la position de plusieurs seuils utiles à l'identification de la classe moyenne de revenu. Il apparaît que cette distribution est très oblique à gauche, traduisant le haut niveau de pauvreté dans le pays. Le revenu mensuel par tête médian est de 15000 FCFA, soit un niveau très proche du seuil de pauvreté de 2 \$ par tête par jour, en PPA. Les différents intervalles possibles en fonction des trois approches décrites plus haut produisent des résultats très disparates en termes de poids de la classe moyenne de revenu, celui-ci allant de moins de 2 pour cent à plus de 46 pour cent (tableau A2, annexé en ligne). Cette grande disparité pose évidemment la question du choix de l'intervalle le plus pertinent. Au regard de la littérature et de nos résultats, notre choix s'est porté sur une approche mixte. Nous avons ainsi retenu le seuil de $4 \$$ comme borne inférieure et le $95^{\mathrm{e}}$ percentile de la distribution des revenus comme borne supérieure afin d'exclure les 5 pour cent les plus riches de la population. ${ }^{6}$ Le choix de cette borne inférieure nous distingue de l'approche dominante qui suppose qu'il suffit de ne pas être pauvre pour appartenir à la classe moyenne. La littérature existante et les travaux récents sur cette question soulignent pourtant que les ménages échappant à la pauvreté grâce à un revenu par tête situé entre 2 \$ et 4 \$ par jour (la "classe flottante " de la BAD) restent fortement vulnérables à des retours fréquents, nombreux et de durée significative dans la pauvreté en cas de chocs socio-économiques ou de simples aléas personnels (Kroeker, O’Kane, et Scharrer 2018; Melber 2016). C’est afin de s'éloigner de toute 


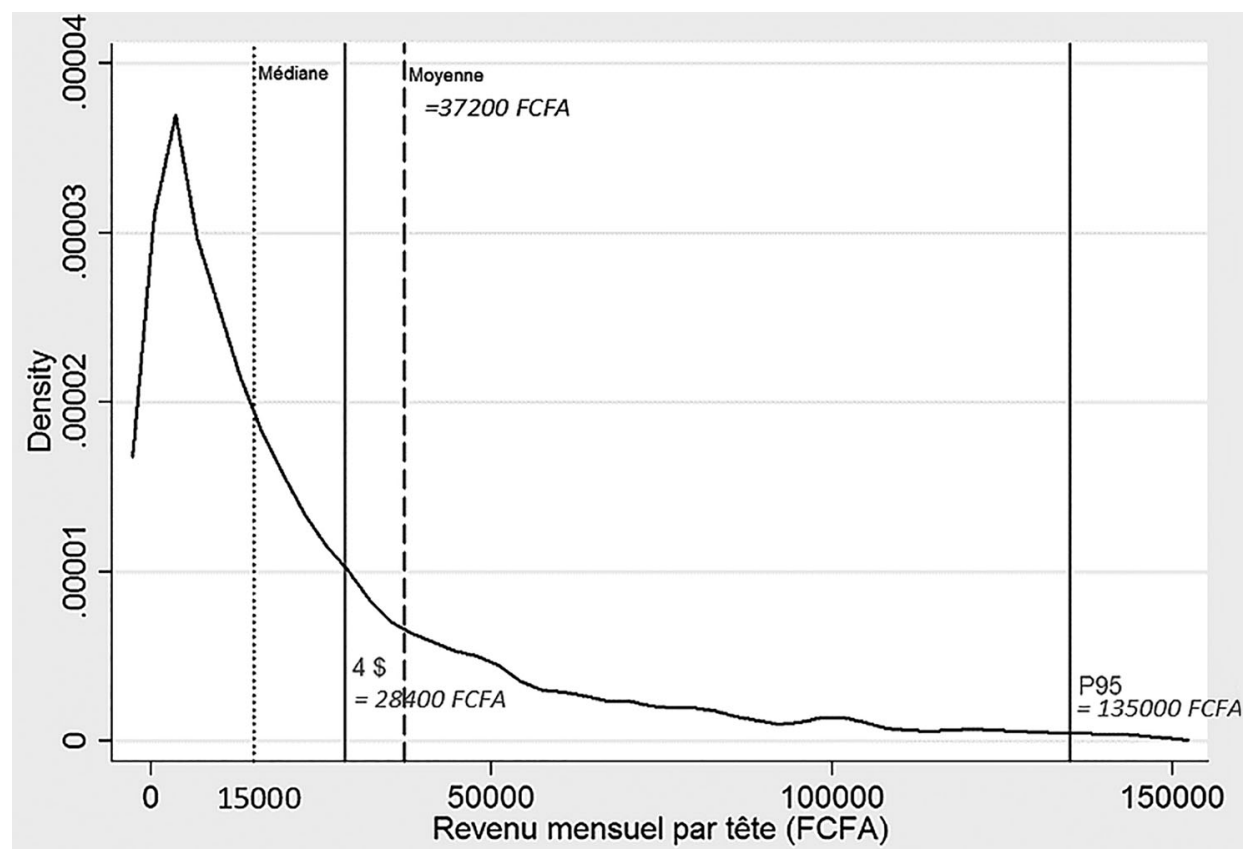

Graphique 1. Distribution des revenus mensuels par tête en FCFA (Côte d'Ivoire, 2015). Source : ENV (2015); calcul des auteurs.

évidence du seuil de pauvreté de 2 \$ par jour, au-dessous duquel se situe près de la moitié de la population, que nous avons opté pour celui de $4 \$$, soit le double.

Ainsi calibrée, la classe moyenne pécuniaire ivoirienne, « les gens du milieu » de la distribution des revenus, représente finalement une masse relativement limitée de 26,4 pour cent de la population. ${ }^{7}$ La taille moyenne des ménages de cette classe étant de 3 personnes (voir plus haut), on obtient un revenu mensuel moyen par ménage approximativement compris entre 85200 FCFA (360 \$) et 405000 FCFA (1710\$).

Cette classe moyenne ivoirienne présente quelques caractéristiques globales intéressantes, notamment au regard de nos variables de classification retenues (Tableau 1). Ainsi, 48,1 pour cent des chefs de ménage de cette catégorie sont sans formation. Un résultat qui contraste quelque peu avec les représentations habituelles d'une classe moyenne ivoirienne essentiellement composée d'individus au niveau de formation secondaire, au minimum (CFAO 2015). Au regard des caractéristiques d'occupation d'emploi, on trouve au sein de cette classe moyenne une proportion importante de chefs de ménage dans les catégories professions intermédiaires, employés et travailleurs des services et ouvriers. Concernant le statut d'emploi, ces chefs de ménage sont majoritairement indépendants ou salariés-employés, et pour 70,6 pour cent d'entre eux, ils exercent dans le secteur informel urbain ou rural. On note que 11 pour cent d'entre eux sont dans la fonction publique, une proportion deux fois moindre que pour les 5 pour cent les plus riches.

D’autres éléments de caractérisation statistique peuvent être relevés à partir du Tableau 2 (annexé en ligne). Les ménages de cette classe moyenne sont majoritairement urbains (à près de $60 \%$ ), mais seuls 16 pour cent vivent à Abidjan. Cela traduit vraisemblablement l'importance des transformations sociales associées aux villes secondaires, tout comme le 
Tableau 1. Caractéristiques (variables de classification) des différents groupes (pauvres, classe moyenne, riche), Côte d'Ivoire, 2015.

\begin{tabular}{|c|c|c|c|c|}
\hline & Pauvres & Classe moyenne & Riches & Ensemble \\
\hline \multicolumn{5}{|l|}{ Éducation } \\
\hline Sans éducation & 66,0 & 48,1 & 33,4 & 59,6 \\
\hline Éducation primaire & 16,5 & 16,6 & 13,1 & 16,3 \\
\hline Éducation secondaire & 16,2 & 27,6 & 27,5 & 19,8 \\
\hline Éducation supérieure & 1,4 & 7,8 & 26,1 & 4,3 \\
\hline \multicolumn{5}{|l|}{ Catégorie socioprofessionnelle } \\
\hline Inactifs et chômeurs & 38,7 & 15,2 & 11,4 & 31,2 \\
\hline Dirigeants, cadres supérieurs, professions intellectuelles & 0,8 & 4,3 & 13,4 & 2,4 \\
\hline Professionnels intermédiaires & 3,1 & 12,1 & 15,8 & 6,1 \\
\hline Employés et travailleurs des services & 10,9 & 20,6 & 22,9 & 14,0 \\
\hline Ouvriers & 13,9 & 21,0 & 15,2 & 15,8 \\
\hline Agriculteurs & 32,6 & 26,9 & 21,3 & 30,5 \\
\hline \multicolumn{5}{|l|}{ Statut dans l'emploi } \\
\hline Inactifs et chômeurs & 37,6 & 14,5 & 11,0 & 30,2 \\
\hline Salariés, employés & 15,5 & 38,1 & 48,3 & 23,1 \\
\hline Indépendants & 38,9 & 43,0 & 36,1 & 39,8 \\
\hline Employeurs & 0,4 & 1,1 & 1,9 & 0,6 \\
\hline Travailleurs non rémunérés & 7,7 & 3,3 & 2,8 & 6,3 \\
\hline \multicolumn{5}{|l|}{ Type d'employeur, secteur institutionnel } \\
\hline Inactifs et chômeurs & 37,6 & 14,5 & 11,0 & 30,2 \\
\hline Emploi privé formel & 1,3 & 3,8 & 8,1 & 2,3 \\
\hline Emploi privé informel & 59,0 & 70,6 & 58,1 & 62,0 \\
\hline Emploi public & 2,1 & 11,1 & 22,8 & 5,5 \\
\hline \multicolumn{5}{|l|}{ Pluriactivité } \\
\hline Non & 97,4 & 96,1 & 91,5 & 96,8 \\
\hline Oui & 2,6 & 3,9 & 8,5 & 3,2 \\
\hline
\end{tabular}

Note : La classe moyenne est définie comme les ménages dont le revenu se situe entre $4 \$$ par jour et le $95^{\mathrm{e}}$ percentile. Source : ENV (2015); calcul des auteurs.

haut niveau d'inégalités qui caractérise la capitale ivoirienne, avec une forte concentration des richesses dans le 5 pour cent du haut des revenus et une masse importante de la population en situation de précarité. Ce sentiment d'exacerbation des inégalités à Abidjan est assez largement confirmé par l'ensemble des personnes interrogées lors des entretiens qualitatifs. On remarque d'ailleurs que, si en moyenne 46 pour cent des chefs de ménage de la classe moyenne sont propriétaires de leur logement, cette proportion est tirée vers le haut par les classes moyennes rurales, alors qu'en milieu urbain, on se rapproche du taux de 34 pour cent estimé par UCT-UNILEVER (2017) pour Abidjan. Le taux d'épargne dans l'ensemble de cette classe est en moyenne de 10 pour cent du revenu du ménage.

Les entretiens qualitatifs ont également permis d'approfondir ces constats et de préciser certains comportements et aspirations communs des classes moyennes. ${ }^{8}$ On trouve ainsi, dans l'ensemble des entretiens, un effort d'épargne assez conséquent. Comme le souligne M. B., chef de service à la poste : "Quand je n'ai pas de soucis particuliers, je peux mettre entre 20 pour cent et 30 pour cent de mon salaire à l'épargne » (entretien $n^{\circ} 3$ ). Cet effort d'épargne se réalise dans des circuits financiers formels comme informels. À cet égard, plusieurs entretiens ont souligné le rôle du mobile money comme nouvelle occasion d'« épargne légère ». À côté de cet effort d'épargne, les entretiens révèlent aussi un effort commun à l'ensemble des ménages de cette catégorie envers l'éducation des enfants, qui apparaît comme une de leurs préoccupations majeures. Cela les conduit d'ailleurs à opter bien souvent pour le secteur privé dès que le secteur public est défaillant : "l'école est le parent pauvre de la Côte d'Ivoire! ", selon l'un des interrogés. De manière transversale, on note une dénonciation de l'accroissement des inégalités et de la corruption 
Tableau 2. Distributions comparées des variables de classification selon la classe, Côte d'Ivoire, 2015.

\begin{tabular}{|c|c|c|c|c|c|c|}
\hline & $\begin{array}{c}\text { Classe } 1 \\
(25 \%)\end{array}$ & $\begin{array}{c}\text { Classe } 2 \\
(39 \%)\end{array}$ & $\begin{array}{c}\text { Classe } 3 \\
(4 \%)\end{array}$ & $\begin{array}{c}\text { Classe } 4 \\
(17 \%)\end{array}$ & $\begin{array}{c}\text { Classe } 5 \\
(15 \%)\end{array}$ & Ensemble \\
\hline \multicolumn{7}{|l|}{ Statut du chef dans l'emploi } \\
\hline Sans emploi (inactifs, chômeurs) & 0,0 & 0,0 & 0,0 & 0,0 & 100,0 & 14,5 \\
\hline Salariés/Employés & 1,2 & 46,2 & 87,6 & 94,9 & 0,0 & 38,1 \\
\hline Indépendants & 97,9 & 45,4 & 10,9 & 0,5 & 0,0 & 43,0 \\
\hline Employeurs & 0,4 & 1,4 & 0,0 & 2,9 & 0,0 & 1,1 \\
\hline Travailleurs non rémunérés & 0,6 & 7,1 & 1,6 & 1,7 & 0,0 & 3,3 \\
\hline \multicolumn{7}{|l|}{ Catégorie socioprofessionnelle } \\
\hline Sans emploi (inactifs, chômeurs) & 0,0 & 0,0 & 0,0 & 0,0 & 100,0 & 14,5 \\
\hline Dirigeants/Cadres supérieurs & 0,4 & 1,4 & 8,5 & 18,2 & 0,0 & 4,1 \\
\hline Professionnels intermédiaires & 0,0 & 0,5 & 34,9 & 58,3 & 0,0 & 11,6 \\
\hline Employés et travailleurs des services & 1,9 & 50,8 & 26,4 & 16,2 & 0,0 & 24,2 \\
\hline Ouvriers & 0,4 & 45,6 & 26,4 & 6,0 & 0,0 & 20,0 \\
\hline Agriculteurs & 97,4 & 1,8 & 3,9 & 1,4 & 0,0 & 25,7 \\
\hline \multicolumn{7}{|l|}{ Employeur secteur institutionnel } \\
\hline Sans emploi (inactifs, chômeurs) & 0,0 & 0,0 & 0,0 & 0,0 & 100,0 & 14,5 \\
\hline Emploi privé formel & 0,0 & 0,0 & 100,0 & 0,0 & 0,0 & 3,8 \\
\hline Emploi privé informel & 99,8 & 99,3 & 0,0 & 37,5 & 0,0 & 70,6 \\
\hline Emploi public & 0,2 & 0,7 & 0,0 & 62,5 & 0,0 & 11,1 \\
\hline \multicolumn{7}{|l|}{ Éducation du chef de ménage } \\
\hline Sans éducation & 64,5 & 54,7 & 20,2 & 16,7 & 48,9 & 48,5 \\
\hline Éducation primaire & 19,7 & 19,8 & 10,1 & 8,2 & 13,0 & 16,4 \\
\hline Éducation secondaire & 15,2 & 23,9 & 51,9 & 44,6 & 31,4 & 27,4 \\
\hline Éducation supérieure & 0,7 & 1,6 & 17,8 & 30,5 & 6,7 & 7,7 \\
\hline \multicolumn{7}{|l|}{ Pluriactivité } \\
\hline Monoactivité & 92,2 & 97,3 & 98,5 & 96,8 & 98,2 & 96,1 \\
\hline Pluriactivité & 7,8 & 2,7 & 1,6 & 3,2 & 1,8 & 3,9 \\
\hline
\end{tabular}

Note : Les cases en gras dans le tableau ciblent les modalités qui, d'un point de vue statistique, sont significativement surreprésentées dans la classe considérée par rapport au reste de la population (test sur résidu ajustés standardisés du Khi-2, sig.<0,05).

Source : ENV (2015); calcul des auteurs.

des élites, caractérisant une "Côte d'Ivoire à deux vitesses ». L'écart entre les discours sur la croissance à deux chiffres et les conditions de la majorité qui ne cessent de se détériorer est souvent dénoncé : "Une classe roule avec des voitures à 50 millions de FCFA pendant que les gens s'appauvrissent. Les injustices sont trop criantes! » (M. H, entretien $\left.\mathrm{n}^{\mathrm{o}} 27\right)$. Pour autant, cela ne se traduit pas par un engagement politique marqué : nos entretiens des ménages montrent que ces classes moyennes sont essentiellement mues par la recherche d'intérêts personnels assez matériels (conditions de vie, logement, sécurité ...) et par la protection de leurs stratégies de promotion personnelle et familiale. Ces classes se caractérisent de manière générale surtout par un positionnement individualiste éloigné de toute forme d'identité collective, en se tenant à l'écart de l'État et des enjeux politiques.

$\mathrm{Au}$-delà de ces quelques marqueurs forts d'appartenance à la classe moyenne ivoirienne, il s'agit maintenant d'étudier la cohérence de ce groupe de 26,4 pour cent de la population.

\section{L'hétérogénéité de la classe moyenne ivoirienne : des gens de milieux sociaux différents}

Nous analysons l'hétérogénéité de la classe moyenne de revenu ivoirienne en procédant à la classification multidimensionnelle décrite précédemment. Les résultats de cette 
procédure de classification nous conduisent à identifier cinq groupes distincts les uns des autres et fortement homogènes. Sur la base de l'analyse des distributions comparées des variables de classification (niveau de formation, catégorie socioprofessionnelle, statut d'emploi, type d'employeur et pluriactivité - Tableau 2) et d'un ensemble d'autres variables de caractérisation (Tableau 3 et tableau A5, annexé en ligne), nous pouvons décrire ces cinq groupes. Les résultats des entretiens qualitatifs permettront également de compléter cette description statistique.

D’abord, la classe moyenne ivoirienne des agriculteurs (25\%) est constituée presque exclusivement d'agriculteurs indépendants. En moyenne, les chefs de ces ménages sont relativement âgés et ils disposent des revenus les plus faibles. Toutefois, il faut garder à l'esprit que leur pouvoir d'achat peut rester assez conséquent, compte tenu d'un niveau des prix bien plus faible en milieu rural qu'à Abidjan (notamment dans l'immobilier).

Tableau 3. Distributions et moyennes comparées des variables de caractérisation selon la classe, Côte d'Ivoire 2015.

\begin{tabular}{|c|c|c|c|c|c|c|}
\hline & $\begin{array}{c}\text { Classe } 1 \\
(25 \%)\end{array}$ & $\begin{array}{c}\text { Classe } 2 \\
(39 \%)\end{array}$ & $\begin{array}{c}\text { Classe } 3 \\
(4 \%)\end{array}$ & $\begin{array}{c}\text { Classe } 4 \\
(17 \%)\end{array}$ & $\begin{array}{c}\text { Classe } 5 \\
(15 \%)\end{array}$ & Ensemble \\
\hline \multicolumn{7}{|l|}{ Sexe (\%) } \\
\hline Homme & 91,3 & 82,4 & 93,8 & 86,2 & 71,2 & 84,1 \\
\hline Femme & 8,7 & 17,6 & 6,2 & 13,8 & 28,8 & 15,9 \\
\hline \multicolumn{7}{|l|}{ Statut matrimonial (\%) } \\
\hline Mariage classique & 39,5 & 26,7 & 40,3 & 41,9 & 31,0 & 33,7 \\
\hline Mariage traditionnel ou polygamie & 29,8 & 18,7 & 19,6 & 19,4 & 17,9 & 21,5 \\
\hline Union libre & 5,7 & 6,1 & 15,5 & 11,9 & 6,1 & 7,4 \\
\hline Seul (célibataire, divorcé, veuf) & 24,9 & 48,5 & 25,6 & 26,8 & 45,0 & 37,4 \\
\hline \multicolumn{7}{|l|}{ Nationalité (\%) } \\
\hline Ivoirienne & 72,1 & 67,1 & 85,3 & 89,3 & 83,2 & 75,2 \\
\hline Non ivoirienne & 27,9 & 32,9 & 14,7 & 10,7 & 16,8 & 24,8 \\
\hline \multicolumn{7}{|l|}{ Milieu de résidence (\%) } \\
\hline Abidjan & 1,8 & 20,1 & 37,2 & 25,9 & 14,2 & 16,3 \\
\hline Urbain autre & 23,2 & 45,2 & 43,4 & 53,5 & 47,7 & 41,4 \\
\hline Rural & 75,1 & 34,7 & 19,4 & 20,6 & 38,1 & 42,3 \\
\hline Âge moyen du chef de ménage & 43,1 & 36,2 & 39,9 & 39,6 & 45,3 & 40,0 \\
\hline Taille moyenne du ménage & 2,9 & 2,5 & 3,6 & 3,9 & 2,8 & 2,9 \\
\hline \multicolumn{7}{|l|}{ Revenus, dépenses et épargne (moyennes) } \\
\hline Revenu mensuel ménage (CFA) & 149623 & 132035 & 217197 & 235827 & 148874 & 160041 \\
\hline Revenu mensuel par personne (CFA) & 54778 & 57162 & 62710 & 64699 & 52503 & 57396 \\
\hline Part des dépenses pour l'éducation & 0,004 & 0,013 & 0,034 & 0,032 & 0,030 & 0,017 \\
\hline Part des dépenses pour les communications & 0,045 & 0,066 & 0,078 & 0,071 & 0,065 & 0,062 \\
\hline Part des dépenses pour le transport & 0,104 & 0,105 & 0,098 & 0,118 & 0,090 & 0,104 \\
\hline Part des dépenses pour l'habillement & 0,053 & 0,055 & 0,050 & 0,050 & 0,046 & 0,052 \\
\hline Part des dépenses pour la santé & 0,023 & 0,017 & 0,035 & 0,021 & 0,031 & 0,022 \\
\hline Part des dépenses de transfert & 0,108 & 0,088 & 0,093 & 0,093 & 0,066 & 0,091 \\
\hline Montant annuel d'épargne (FCFA) & 219998 & 150347 & 149242 & 182041 & 97600 & 165697 \\
\hline Taux d'épargne & 0,128 & 0,115 & 0,064 & 0,071 & 0,066 & 0,102 \\
\hline \multicolumn{7}{|l|}{ Actifs possédés (\%) } \\
\hline Possédez-vous une habitation? & 77,0 & 34,2 & 18,6 & 27,9 & 51,5 & 45,8 \\
\hline Possédez-vous un lot non bâti? & 22,4 & 9,0 & 10,9 & 17,6 & 14,8 & 14,8 \\
\hline Possédez-vous des terres agricoles? & 73,40 & 13,40 & 9,30 & 10,60 & 31,60 & 0,46 \\
\hline \multicolumn{7}{|l|}{ Usage des TIC (\%) } \\
\hline Utilisation d'Internet & 1,17 & 6,81 & 36,43 & 28,79 & 8,11 & 10,49 \\
\hline Utilisation d'un ordinateur & 1,05 & 6,21 & 35,66 & 30,83 & 6,69 & 10,34 \\
\hline
\end{tabular}

Note : Les cases en gras dans le tableau ciblent les modalités qui, d'un point de vue statistique, sont significativement surreprésentées (ou dont la moyenne est très supérieure) dans la classe considérée par rapport au reste de la population (test sur résidus standardisés ajustés du Khi-2 ou test t de comparaison des moyennes, sig. $<0,05$ ). Inversement, les cases en italique grisées du tableau montrent les modalités qui, d'un point de vue statistique, sont très sous-représentées (ou dont la moyenne est très inférieure).

Source : ENV (2015); calculs des auteurs. 
Cela s'observe d'ailleurs très bien au plan de leurs actifs puisqu'ils disposent bien plus considérablement que les autres groupes d'une habitation (77\% contre $46 \%$ en moyenne), d'un lot non bâti $(22 \%)$ et, bien sûr, de terres agricoles (73\%). Pour autant, cela ne doit pas masquer la vulnérabilité de leur situation, largement exprimée par les personnes interrogées lors des entretiens qualitatifs, sous l'expression "vivre selon la courbe " (celle $\mathrm{du}$ prix des produits agricoles et du cacao, en particulier). Leurs histoires soulignent en effet la force avec laquelle certains événements influent de manière durable sur leur existence (instabilité des prix et du climat, problèmes de santé, insécurité foncière, etc.).

Ensuite, la classe moyenne des indépendants, employés et ouvriers du secteur informel (39\%) est le plus conséquent des cinq groupes identifiés. Il se distingue des autres par la nature informelle des activités ou des emplois exercés par les chefs de ménage concernés. Derrière l'homogénéité relative à " l'informalité » (le caractère informel de l'activité économique) se cachent en réalité des situations professionnelles quelque peu différenciées. Ainsi, on distingue clairement, selon leur statut professionnel, les indépendants (45\%) des salariés et employés de l'informel (46\%). Une distinction qui se retrouve dans les CSP avec, d'un côté, les ouvriers de l'informel (46\%) et, de l'autre, les employés et travailleurs des services $(51 \%)$. On trouve ces emplois principalement dans les secteurs de l'industrie manufacturière et du commerce. De manière générale, les chefs de ménage de cette catégorie se caractérisent par de nombreux éléments de vulnérabilité. Sur le plan du capital humain, 75 pour cent d'entre eux disposent d'un niveau de formation primaire ou d'aucune formation. Les chefs de ménage de nationalité non ivoirienne sont surreprésentés dans cette catégorie. Ils sont relativement jeunes et vivent seuls pour près de la moitié d'entre eux (leurs ménages sont aussi en moyenne plus petits). Ils disposent du niveau moyen de revenu par ménage le plus faible dans l'ensemble de la classe moyenne et la part de leurs dépenses consacrées à l'éducation et à la santé est particulièrement réduite. Les entretiens qualitatifs ont par ailleurs mis en évidence les conditions de vie difficiles de cette catégorie. Mme A. (entretien $n^{\circ} 28$ ), âgée de 38 ans et gérante d'un salon de coiffure à Cocody, illustre bien cette situation. Elle s'identifie comme étant moins pauvre que les pauvres, mais très éloignée des riches, avec des conditions de vie quotidienne dans l'ensemble difficiles. Ses besoins en matière de logement, de santé et d'éducation ne sont pas pleinement comblés. Elle souhaite cependant conserver un certain optimisme face à l'avenir. Nourrissant des projets futurs où elle serait à son compte, elle veille à épargner un peu. Issue d'un milieu économique rural et difficile, elle pense vivre une petite ascension sociale, bien que celle-ci reste limitée et en deçà de ses ambitions.

Troisièmement, la classe moyenne des salariés du secteur privé formel (4\%) rassemble des salariés et employés du secteur privé formel qui exercent en tant qu'ouvriers, employés et professionnels intermédiaires. On les trouve dans presque toutes les activités, mais ils sont surreprésentés dans les activités immobilières et financières. Par ailleurs, près de 40 pour cent d'entre eux exercent à Abidjan, contre seulement 16 pour cent de l'ensemble des ménages de la classe moyenne. Leurs revenus moyens sont parmi les plus élevés (avec ceux de la catégorie suivante). Ils sont plus connectés à l'Internet que les autres groupes et se distinguent nettement par la part de leurs revenus allouée à l'éducation, aux communications et à la santé. S'ils sont, moins souvent que les autres, propriétaires de leur logement, cela s'explique très certainement par le prix de l'immobilier de " haut de gamme " à Abidjan. Par ailleurs, comparativement aux 
données quantitatives, nos entretiens qualitatifs ont montré que les individus de cette catégorie disposent de capitaux économiques et sociorelationnels qu'ils mobilisent dans des stratégies de diversification des revenus, d'investissement ou de placement. Ainsi, M. H. (entretien $n^{\circ} 27$ ) a plusieurs projets immobiliers : il a acheté deux terrains, à Bingerville et à la Palmeraie et il a souscrit, avec son épouse, à deux projets immobiliers privés. Si M. H. n'aime pas s'endetter, il a néanmoins contracté un emprunt immobilier pour l'achat de son terrain, la personne qui s'occupait de son dossier à la banque étant une connaissance (il a ainsi pu bénéficier d'un taux à $8 \%$ au lieu de $15 \%$ ). Nos entretiens mettent aussi en évidence un temps plus important consacré aux loisirs et à la fréquentation de grands supermarchés et centres d'achats. Ils soulignent enfin une forme de transmission intergénérationnelle du statut de classe moyenne puisque les parents de ces personnes interrogées ont bien souvent été des fonctionnaires de l'administration publique ivoirienne (douanier, gendarme, instituteurs, techniciens de travaux publics) dans les années 1960-1970.

Quatrièmement, la classe moyenne des professions intermédiaires et supérieures du secteur public et de l'informel (17\%) est particulière, car son unité repose sur le statut et la CSP des chefs des ménage concernés. On y trouve ainsi des salariés/employés occupant essentiellement des postes de professions intermédiaires (près de $60 \%$ ) et de dirigeants et cadres supérieurs $(20 \%)$. La quasi-totalité des salariés du secteur public est dans ce groupe, dont ils représentent 62,5 pour cent (en particulier dans les secteurs de l'administration publique, de l'éducation et de la santé). Mais on y trouve aussi un certain nombre d'employés et de professionnels intermédiaires sans statut formel $(37,5 \%)$, exerçant à des paliers de responsabilité assez importants, mais sous des formes contractuelles non formelles. On trouve ces derniers dans les secteurs de l'industrie, de la construction, de l'immobilier et du commerce. Dans l'ensemble, les chefs de ménage de cette catégorie se distinguent par leur haut niveau d'éducation et sont surreprésentés de manière générale en ville, en particulier à Abidjan. Les chefs de ces ménages disposent des revenus mensuels par tête les plus élevés de tous les groupes. À l'instar du groupe précédent, ils consacrent une part plus importante de leur revenu aux dépenses d'éducation. Il ressort des entretiens qualitatifs que les aspirations des personnes interrogées sont assez nettement marquées par une volonté de promotion sociale et de prise en charge de leur destin, incluant une aspiration au statut de riche. On trouve ici des stratégies d'investissement et de diversification des revenus, notamment à travers le statut d'agriculteur « absentéiste » (Affou Yapi 1987).

Cinquièmement, la classe moyenne des retraités et inactifs ${ }^{9}(15 \%)$ regroupe principalement les ménages dirigés par des retraités ou des inactifs. Ces ménages sont plus souvent que ceux des autres groupes dirigés par des femmes. Si leurs revenus sont en moyenne les plus faibles de la classe moyenne, ils disposent en revanche de certains actifs (logement, terres agricoles). Les entretiens qualitatifs ont plutôt révélé des formes de vulnérabilité chez ces ménages qui dépendent beaucoup des transferts de revenus de la part de membres de la famille. Un sentiment de déclassement semble également palpable, comme l'exprime M. J. (entretien $\mathrm{n}^{\mathrm{o}} 31$ ). Préretraité de 64 ans, ancien professeur au collège et au lycée, ayant gravi les échelons de l'administration publique, il affirme qu'auparavant, il se sentait appartenir à une classe moyenne, avec une identité liée à la classe moyenne, mais que depuis 10 ans, il est sorti de cette classe, s'approchant davantage de la pauvreté. 


\section{Continuités historiques et enjeux socioéconomiques contemporains des classes moyennes}

\section{Les héritiers historiques du modèle houphouëtien}

Parmi les catégories constitutives de la classe moyenne ivoirienne, nous trouvons des "piliers historiques » du régime de croissance des années 1960 à 1970, qui semblent bénéficier du renouveau économique que connaît la Côte d'Ivoire depuis 2011.

D'un côté, représentant 25 pour cent de la classe moyenne, les agriculteurs, après des décennies de déclassement, semblent avoir profité de cet environnement plus favorable. Dans un contexte d'urbanisation rapide, il est, de plus, possible que la baisse du poids des agriculteurs conduise à une concentration foncière plus accrue faisant émerger de "gros planteurs" dans des secteurs anciens (cacao) ou plus récents (anacarde, hévéa). Il est pour l'instant difficile de se prononcer catégoriquement là-dessus, d'autant plus que nos statistiques et nos entretiens soulignent plutôt la vulnérabilité de ce groupe, qui connaît des niveaux de revenus assez bas et qui se sent un peu délaissé par les pouvoirs publics. Les enjeux politiques et économiques associés à cette catégorie portent alors sur la sécurisation de leurs conditions de vie face aux différents risques auxquels ils sont confrontés : instabilité des prix, insécurité et tensions foncières, fortes inégalités dans la transmission intergénérationnelle.

De l'autre côté, on trouve les salariés du secteur formel, privé et public, qui se situent aux niveaux les plus élevés des revenus de la classe moyenne. En dépit de la crise, ces catégories semblent avoir pu maintenir leur situation socioéconomique. Il est par ailleurs très probable qu'elles retrouvent le chemin de l'enrichissement sur des bases assez similaires à celle de la période faste grâce à des politiques publiques qui leur sont favorables (doublement du SMIG, hausse des emplois dans la fonction publique, politique d'accès au logement) et à des stratégies individuelles fondées sur l'enchevêtrement des positions dans différents espaces (administratif, politique, économique) par des investissements dans le foncier urbain et agricole, notamment (Colin et Tarouth 2017).

Si ces catégories sont bien des héritières historiques du modèle houphouëtien des années 1960 et 1970 (au sens de successeurs temporels et structurels dans un modèle de croissance), il est difficile d'affirmer que les ménages qui les composent sont aussi des héritiers au sens sociologique du terme (au sens de succession ou de reproduction sociale des positions). En l'absence d'informations sur le statut des parents dans la base de données ENV 2015, nous ne pouvons le prouver. Dans notre étude statistique, nous identifions un groupe de retraités et inactifs particulièrement important (15\%). Ce groupe pourrait exprimer la permanence des groupes de classe moyenne en expansion des années 19601980, mais dont les niveaux de revenus sont limités, et pourrait constituer une des expressions des capacités de transfert intergénérationnel des statuts sociaux. Nos entretiens qualitatifs, quant à eux, confirment bien des trajectoires marquées par des formes d'héritage des positions sociales des parents, en particulier dans les catégories de salariés du formel. Mais on observe aussi, à travers quelques entretiens, des trajectoires d'ascension sociale passant uniquement par le diplôme. Mme C. (entretien $\mathrm{n}^{\mathrm{o}} 29$ ), 37 ans et fonctionnaire (conseillère d'orientation), de père artisan et de mère ménagère, explique avoir pu accéder à la fonction publique par ses propres moyens, grâce à un « système méritocratique fonctionnel ». 
Pour autant, le fait qu'il y ait seulement 4 pour cent de la classe moyenne dans le secteur privé formel et moins de 17 pour cent dans le public traduit bien toutes les problématiques relatives à la transformation structurelle et à la diversification du modèle de croissance ivoirien visant à accroître le nombre d'emplois dans le secteur privé (Banque mondiale 2015). Ce modèle ne semble pas en mesure d'intensifier le processus d'accès direct à la classe moyenne pour les diplômés.

\section{L'informel urbain comme principal mode d'ascension sociale en Côte d'lvoire?}

Dans ce contexte, on observe que le secteur informel et, en particulier, l'informel urbain se révèlent être la principale catégorie constitutive de la classe moyenne ivoirienne (plus de $40 \%)$. La littérature et les débats autour de l'informel en Afrique sont anciens. Derrière cette catégorie ou variable statistique "informel " se cache en réalité une grande diversité de situations d'emplois, d'activités, de statut social, de revenu, etc. Nos résultats confirment cette forte hétérogénéité de l'informel en Côte d'Ivoire. Au sein de la classe moyenne ivoirienne, nous trouvons les indépendants, employés et ouvriers du secteur informel (39\%) chez lesquels les situations sont relativement contrastées, notamment entre indépendants, d'un côté, et ouvriers, de l'autre. Mais nous observons aussi des employés informels au sein de la catégorie des professions intermédiaires et supérieures, à des niveaux de revenus moyens bien plus élevés. Ainsi, nous trouvons l'informel urbain aux différents étages de revenus de la classe moyenne. Un résultat cohérent avec d'autres travaux qui montrent qu'en Côte d'Ivoire, ce sont désormais aussi les personnes du secteur informel urbains qui constituent la fraction majoritaire des détenteurs de hauts revenus, c'est-à-dire des 1 pour cent les plus riches (47\% en 2014 contre $27 \%$ en 1998 Cogneau, Czajka, et Houngbedji 2017). Ces résultats traduisent la forte « informalisation » du marché du travail en Côte d'Ivoire, en même temps qu'ils soulignent très probablement l'existence d'un flux de "néo » classes moyennes qui émergent plutôt par le bas et restent assez vulnérables.

Ce constat soulève d'importants enjeux tant économiques que sociopolitiques. Sur le plan économique, il en va par exemple de la capacité de ces activités informelles à s'inscrire dans une logique d'intensification dans un projet global d'industrialisation et d'émergence du pays. Il est probable que, parmi ces indépendants et leurs microentreprises, on trouve les fameuses " gazelles contraintes » identifiées par Grimm, Knorringa, et Lay (2012). On peut en effet aisément considérer que le sens de l'entrepreneuriat de cette catégorie des classes moyennes est bien présent, ne serait-ce que dans sa capacité à jouer de la pluriactivité dans une logique d'accumulation extensive. Seulement, le fort potentiel de ces entreprises se voit limité par un environnement particulièrement instable et contraint. L'objectif des politiques publiques consiste alors à lever les contraintes auxquelles font face ces microentreprises dans un processus graduel et équilibré maniant promotion (soutien à l'activité et à l'accroissement de la productivité) et formalisation (fiscalisation et respect des normes de travail). Sur le plan sociopolitique, ce résultat vient nuancer les liens théoriques supposés immédiats entre montée des classes moyennes, d'une part, et d'autre part, renforcement et stabilisation des institutions (bonne gouvernance, démocratie, etc. - Giesbert and Schotte 2016). Le secteur informel se construit en effet pour partie en dehors de l'État, voire contre celui-ci. Par ailleurs, le poids considérable de l'informel urbain dans la classe moyenne ivoirienne participe évidemment de sa faible capacité de 
mobilisation politique. Le dualisme formel/informel qui traverse cette classe moyenne contribue à alimenter des conflits et des différences dans les attentes concernant les politiques publiques (notamment sur les questions de fiscalité et de redistribution).

\section{Conclusion}

En conclusion, cette analyse approfondie de la composition des classes moyennes ivoiriennes contemporaines, de leurs caractéristiques et comportements, nous permet de tirer les quatre enseignements principaux suivants.

Premièrement, aujourd'hui en Côte d'Ivoire, l'ampleur de la classe moyenne de revenu telle que nous l'identifions ( 4 \$ - P95) est limitée à 26 pour cent de la population. Dans cet ensemble, on peut reconnaître des traits communs : un goût partagé pour l'effort sur soi et pour la promotion de soi; une même importance accordée à l'éducation des enfants; un même effort soutenu d'épargne; un même positionnement très individualiste et matérialiste, une absence d'identification commune à la classe moyenne.

Deuxièmement, cette classe moyenne ivoirienne est particulièrement hétérogène quant aux catégories socioprofessionnelles, à l'éducation et aux comportements. Nos résultats soulignent qu'elle est constituée de cinq groupes distincts : (1) les agriculteurs; (2) les indépendants, employés et ouvriers du secteur informel; (3) les salariés du secteur privé formel; (4) les personnes ayant des professions intermédiaires et supérieures des secteurs public et informel; et (5) les retraités ou inactifs.

Troisièmement, parmi ces cinq groupes, nous trouvons des héritiers historiques du modèle houphoüetien des années 1960-1970, à savoir les agriculteurs, d'un côté, et les salariés du secteur formel (privé et public), de l'autre. Seulement, le contexte a changé. Et si les voies d'enrichissement de ces catégories peuvent à certains égards ressembler à celles de la période du miracle, les enjeux politiques et économiques sont quelque peu différents pour chacune d'entre elles. Mais, plus encore, le changement de contexte est manifeste dans le fait que ces piliers historiques représentent aujourd'hui une part minoritaire de la classe moyenne ivoirienne.

Quatrièmement, l'informel urbain semble devenu la voie principale d'ascension sociale dans le pays, ce qui soulève des enjeux majeurs tant sur le plan économique (dynamiques entrepreneuriales et logiques d'intensification) que sociopolitique (divergence d'intérêts et d'attentes au sein de la classe moyenne, faible capacité de mobilisation politique).

Pour terminer, nos résultats montrent qu'en Côte d'Ivoire, ce que l'on appelle classes moyennes en 2015 recouvre cinq strates marquées par un fort dualisme quant au revenu et à la vulnérabilité. Dès lors, d'un point de vue dynamique, il est important de s'interroger sur le devenir de ce dualisme au sein des classes moyennes ivoiriennes. Est-il en train de se réduire par une massification par le bas et une stabilisation accrue des classes moyennes? Ou traduit-il un creusement des écarts entre les plus riches et le reste de la population et, ainsi, une polarisation de la société ivoirienne?

\section{Notes}

1. Dans ce travail, nous utilisons plus volontiers l'expression " classes moyennes » au pluriel, car notre propos est justement de montrer leur hétérogénéité interne (relativement aux situations socioprofessionnelles, mais aussi aux comportements et situations socioéconomiques). Le 
terme "classe moyenne » au singulier est parfois repris, mais plus par mesure de facilité et comme synonyme de groupe à revenu intermédiaire sans lui donner de signification sociale prédéfinie (mais en retenant qu'il y a derrière cette étiquette plus qu'un phénomène statistique).

2. Si l'enquête couvre différents aspects concernant les conditions de vie des ménages (caractéristiques socioéconomiques, éducation, emploi, différentes sources de revenus, dépenses, actifs, etc.) qui sont bien décrits dans le rapport ENV (2015), nous ne disposions pas dans le cadre de ce projet des modules relatifs aux dépenses alimentaires (empêchant toute mesure du niveau de vie par les dépenses de consommation).

3. Cette variable de revenu a été construite par nos soins à partir des données brutes de l'ENV et après nettoyage des points aberrants. Nous avons testé la robustesse de nos résultats avec une mesure du revenu des ménages par équivalent adulte à partir de l'échelle d'équivalence d'Oxford (chef de ménage $=1$, autre(s) adulte $(s)=0,7$ et enfants $(<14$ ans $)=0,5)$. De manière assez attendue, cela conduit à déplacer la distribution vers la droite et donc à grossir légèrement la classe moyenne par le bas. En effet, 981 ménages pauvres sont reclassés dans la " classe moyenne » par cette méthode (on compte également quelques rares ménages de " classes moyennes » qui deviennent riches et inversement, 74 et 76 respectivement). Au regard des caractéristiques socioprofessionnelles de ces ménages pauvres " promus » vers la classe moyenne, nous trouvons que, pour moitié d'entre eux, ce sont des agriculteurs, l'autre moitié se partageant équitablement entre ouvriers et inactifs. Ainsi, en presque totalité, les personnes actives promues à la classe moyenne avec l'approche en équivalent adulte sont des agriculteurs et des indépendants informels sans formation (ou primaire pour quelques-uns) qui viennent grossir le bas de la classe moyenne. L'approche par le revenu en équivalent adulte ne modifie pas nos résultats concernant la structure globale de notre classe moyenne et, de fait, les enjeux de politique qui lui sont associés (voir plus haut.

4. Le critère d'agrégation de Ward est retenu ici. La partition que nous choisissons (le nombre de groupes retenus au sein de la classe moyenne de revenu) résulte de l'analyse de l'histogramme des indices de niveau d'agrégation des nœuds de la hiérarchie et de l'analyse du dendrogramme.

5. Au-delà de la trajectoire socioéconomique et des conditions de vie actuelles des personnes interrogées (emplois, comportements de consommation, participation à la vie sociale et politique), une attention particulière était portée durant l'entrevue à leurs attentes et aspirations dans différents domaines de politiques publiques (éducation, santé, transport, protection sociale, justice, sécurité).

6. Le tableau A3 présente sur quelques indicateurs (revenus de la propriété, épargne, revenu mensuel par tête du ménage, loyers reçus, etc.) les différences de moyenne entre les 5 pour cent les plus riches de la distribution totale des revenus (P95), les 5 pour cent les plus riches de la classe moyenne (P90-P95) et les 5 pour cent les plus riches suivants (P85P90). Cela confirme l'existence de sauts qualitatifs et quantitatifs assez nets dans la distribution de ces variables entre les 5 pour cent les plus riches et le haut de la classe moyenne (P90-P95).

7. Un résultat finalement assez compatible avec celui obtenu par Ncube et Lufumpa (2015), avec une mesure monétaire reposant sur la dépense de consommation par tête (une classe moyenne ivoirienne représentant 55 pour cent de la population, mais dont 60 pour cent se situent entre $2 \$$ et $4 \$$, hors de notre intervalle).

8. Une synthèse des principales informations issues de l'analyse des entretiens qualitatifs est présentée dans le tableau A4, Annexe en ligne.

9. Les inactifs sont par convention les personnes qui ne sont ni en emploi (BIT) ni au chômage : jeunes de moins de 15 ans, étudiants et retraités ne travaillant pas en complément de leurs études ou de leur retraite, hommes et femmes au foyer, personnes en incapacité de travailler (Institut national de la statistique et des études économiques-Insee). https://www.insee.fr/fr/ metadonnees/definition/c2018. 


\section{Remerciements}

Nous remercions l'Institut national de la statistique de Côte d'Ivoire pour la mise à disposition des données de l’Enquête sur les conditions de vie des ménages de 2015 (ENV 2015).

\section{Financement}

Cet article est issu d'un projet de recherche comparative financé par l'Agence française de développement (AFD) sur «Les implications de l'essor des classes moyennes dans les pays en développement et émergents » et portant sur quatre pays distincts : le Brésil, la Côte d'Ivoire, la Turquie et le Vietnam.

\section{Les auteurs}

Jean-Philippe Berrou est actuellement Maître de Conférences en Sciences Economiques à Sciences Po Bordeaux et chercheur au laboratoire LAM (Les Afriques dans le Monde).

Dominique Darbon est actuellement Professeur des Université en Science Politique à Sciences Po Bordeaux et directeur du laboratoire LAM (Les Afriques dans le Monde).

Anne Bekelynck est actuellement monitrice des études en sciences sociales (MES) du programme PAC-CI (site ANRS de Côte d'Ivoire).

Christian Bouquet est actuellement Professeur émérite de géographie politique à l'Université Bordeaux-Montaigne et chercheur au laboratoire LAM (Les Afriques dans le Monde).

Matthieu Clément est actuellement Maître de Conférences en Sciences Economiques à l'Université de Bordeaux et chercheur au laboratoire GREThA (Groupe de Recherche en Economie Théorique et Appliquée).

François Combarnous est actuellement Professeur des Universités en Sciences Economiques à l'Université de Bordeaux et chercheur au laboratoire GREThA (Groupe de Recherche en Economie Théorique et Appliquée).

Éric Rougier est actuellement Maître de Conférences en Sciences Economiques à l'Université de Bordeaux et chercheur au laboratoire GREThA (Groupe de Recherche en Economie Théorique et Appliquée).

\section{Référénces}

Affou Yapi, S. 1987. Les planteurs absentéistes de Côte d'Ivoire. Paris: ORSTOM.

Akindès, F. 2018. «'On ne mange pas les ponts et le goudron' : les sentiers sinueux d'une sortie de crise en Côte d'Ivoire. » Politique Africaine 4 (148): 5-26.

BAD (Banque Africaine de Développement). 2011. "Dynamics of the Middle Class in Africa." Market Brief. http://www.afdb.org.

Banerjee, A., et E. Duflo. 2008. "What is Middle Class about the Middle Classes around the World? » Journal of Economic Perspectives 22 (2): 3-28.

Banque Mondiale. 2015. La Force de l'Eléphant. Pour que sa croissance génère plus d'emploi de qualité. Rapport Situation Economique Côte d'Ivoire. Washington, DC : Banque Mondiale.

Birdsall, N. 2010. " The (Indispensable) Middle Cass in Developing Countries. » Dans Equity and Growth in a Globalizing World, sous la direction de R. Kanbur et M. Spence, 157-187. Washington DC: World Bank.

Bonnefond, C., M. Clément, et F. Combarnous. 2015. « In Search of the Elusive Chinese Urban Middle Class : An Exploratory Analysis. » Post-Communist Economies 27 (1): 41-59.

CFAO. 2015. Les classes moyennes en Afrique, quelle réalité ? Quels enjeux ? http://www.cfaogroup. com. 
Cogneau, D., L. Czajka, et K. Houngbedji. 2017. « Le retour de l'éléphant triomphant? Croissance et inégalités de revenu en Côte d'Ivoire (1988-2015). » Afrique contemporaine 263-264 (3): 221225.

Cogneau, D., et S. Mesplé-Somps. 2003. «Les illusions perdues de l'économie ivoirienne et la crise politique. " Afrique contemporaine 206 (2): 87-104.

Colin, J. P., et H. G. Tarouth. 2017. « Les élites urbaines comme nouveaux acteurs du marché foncier en Côte d'Ivoire. » Géographie, économie, société 19 (3): 331-355.

Contamin, B., et H. Memel-Foté, dirs. 1997. Le modèle ivoirien en questions. Crise, ajustements, recompositions. Paris: Karthala-ORSTOM.

Darbon, D. 2012. «Classe(s) moyenne(s) : une revue de la littérature. Un concept utile pour suivre les dynamiques de l'Afrique. " Afrique contemporaine 244 (4): 33-51.

Easterly, W. 2001. "The Middle Class Consensus and Economic Development. " Journal of Economic Growth 6 (4): 317-335.

ENV. 2015. Enquête sur les conditions de vie des ménages en Côte d'Ivoire - 2015. Abidjan: Institut National de la Statistique.

Fauré, Y.-A., et J.-F. Médard, dirs. 1982. Etat et bourgeoisie en Côte d'Ivoire. Paris: Karthala.

Giesbert, L., et S. Schotte. 2016. «Africa's New Middle Class : Fact and Fiction of Its Transformative Power. » GIGA Focus 1 (May 2016). ISSN 1862-3603.

Grimm, M., C. Guénard, et S. Mesplé-Somps. 2002. «What Has Happened to the Urban Population in Côte d'Ivoire since the 1980s? An Analysis of Monetary Poverty and Deprivation over 15 Years of Household Data. " World Development 30 (6): 1073-1095.

Grimm, M., P. Knorringa, et J. Lay. 2012. «Informal Entrepreneurs in Western Africa: Constrained Gazelles in the lower Tier. » World Development 40 (7): 1352-1368.

Huntington, S. 1968. Political Order in Changing Societies. Yale: Yale University Press.

Kroeker, L., D. O'Kane, et T. Scharrer, dirs. 2018. Middle Classes in Africa: Changing Lives and Conceptual Challenges. London: Palgrave Macmillan.

Le Pape, M. 1997. L'énergie sociale à Abidjan : économie politique de la ville en Afrique noire, 1930-1945. Paris: Karthala.

Melber, H., dir. 2016. The Rise of Africa's Middle Class. London: Zed Books.

Nallet, C. 2018. «De nouveaux lieux de consommation pour de nouvelles classes moyennes? Les centres commerciaux à Abidjan. » Notes de l'Ifri. Paris : Institut français des relations internationales (Ifri).

Ncube, M., et C. L. Lufumpa, dirs. 2015. The Emerging Middle Class in Africa. London: Routledge.

OCDE. 2016. Examen multidimensionnel de la Côte d'Ivoire. Volume 1 : Évaluation initiale. Paris: OCDE.

Savina, A. M., P. Antoine, A. Dubresson, et D. A. Yapi. 1985. "'Les en-haut des en-bas et les en-bas des en-haut'. Classes moyennes et urbanisation à Abidjan (Côte d'Ivoire). » Tiers-Monde 26 (101): 55-68.

Thurlow, J., D. Resnick, et D. Ubogu. 2015. « Matching Concept with Measurement: Who Belongs to Africa’s Middle Class? » Journal of International Development 27: 588-608.

UCT-Unilever Institute of Strategic Marketing. 2017. The African Middle Class: Who Are They? Rapport disponible sur: https://www.ipsos.com/en-za/african-lions-charting-rise-middle-classsub-saharan-africa. 